\title{
Delineamento de Tarefas de Cálculo Diferencial e Integral Envolvendo Sequências Numéricas: Análise de um Processo
}

\section{Delineation of Differential and Integral Calculation Tasks involving Numerical Sequences: analysis of a process}

\author{
Nélvia Santana Ramos a ; André Luis Trevisan"; Marcele Tavares Mendes ${ }^{\mathrm{a}}$ \\ a Universidade Tecnológica Federal do Paraná, Londrina, Brasil- nelvialramos@gmail.com, andrelt@utfpr.edu.br, \\ marceletavares@utfpr.edu.br
}

\section{Palavras-chave: \\ Pesquisa de desenvolvimento. Cálculo diferencial e integral. Tarefas matemáticas. Sequências numéricas.}

Resumo: Assumindo a importância de refletir acerca do delineamento de tarefas matemáticas, em especial para aulas de Cálculo Diferencial e Integral, intenta-se neste artigo analisar o processo de delineamento de uma das tarefas que compõem o produto educacional oriundo de uma dissertação de mestrado profissional em Ensino de Matemática: como foi elaborada, apresentada/trabalhada com os estudantes e, quando necessário, redesenhada e reaplicada. Para tanto, adotam-se pressupostos da Pesquisa de Desenvolvimento e apresenta-se uma análise de cunho qualitativo, de natureza essencialmente descritiva, incluindo ciclos de aplicação que ocorreram ao longo de quatro semestres, em quatro contextos distintos. Na análise, procuramos evidenciar, por meio do item da tarefa selecionada, (i) elementos resultantes do percurso metodológico de delineamento das tarefas; (ii) fases de seu delineamento e (iii) análise retrospectiva e considerações da professorapesquisadora, primeira autora do texto.
Abstract: Assuming the importance of reflecting on the delineation of mathematical tasks, especially for classes of Differential and Integral Calculus, this article aims to analyze the process of designing one of the tasks that make up the educational product coming from a dissertation of professional masters in Mathematics Teaching: how it was elaborated, presented / worked with the students and, when necessary redesigned and reapplied. In order to do this, the assumptions of the Design Research are adopted and a qualitative and essentially descriptive data analysis is presented, including application cycles that occurred over four semesters in four different contexts. In our analysis, we tried to highlight, through the selected task item: (i) elements resulting from the methodological course of work design; (ii) phases of its design; (iii) retrospective analysis and considerations of the teacherresearcher, the first author of the text. 


\section{Introdução}

Ao longo das últimas décadas, a pesquisa de desenvolvimento (PD), segundo tradução proposta por Matta et al. (2014) e Barbosa e Oliveira (2015), ou investigação baseada em design (IBD) ${ }^{1}$ conforme Ponte et al. (2016), tem-se mostrado uma alternativa para o estudo teórico e empírico da aprendizagem em meio ao complexo processo educacional. A expressão refere-se a uma metodologia de pesquisa que envolve o delineamento e avaliação de artefatos a serem utilizados em um determinado contexto (BARBOSA; OLIVEIRA, 2015). A palavra desenvolvimento refere-se ao processo contínuo de refinamento desse artefato por meio de uma avaliação sistemática.

Segundo Mestre (2014, p. 91-92), a comunidade acadêmica tem reafirmado

o valor desta metodologia, relevando a sua potencialidade para produzir práticas educativas inovadoras. Desta forma, identificam quatro áreas onde este design se torna mais promissor: a) explorar possibilidades de criar novos ambientes de aprendizagem e ensino, b) desenvolver teorias de aprendizagem e ensino que sejam baseadas no contexto, c) avançar e consolidar o conhecimento do design e d) aumentar a capacidade de inovação educacional.

Entendemos que a "vocação para pesquisa aplicada" em educação (MATTA et al., 2014, p. 25), associada à IBD, torna-a uma metodologia promissora em Programas de PósGraduação de cunho profissional, como é o caso do PPGMAT-Mestrado Profissional em Ensino de Matemática da UTFPR, campus Londrina/Cornélio Procópio, ao qual estão vinculados os autores deste texto. Nosso intuito, aqui, é apresentar o processo de delineamento de um artefato para aulas de Cálculo Diferencial e Integral (CDI) 1, de modo restrito, uma tarefa para a exploração de conceitos relacionados a sequências numéricas infinitas, oriunda da dissertação da primeira autora (RAMOS, 2017) ${ }^{2}$.

O objetivo deste artigo justifica-se em função do caráter cíclico da IBD. Intenta-se explicitar como uma das tarefas foi elaborada, apresentada e trabalhada com os estudantes e, quando necessário, redesenhada e reaplicada para que um novo ciclo se iniciasse, o que pode ser um meio de favorecer a reflexão a respeito do ambiente de ensino e de aprendizagem de CDI. A análise aqui apresentada é de natureza essencialmente descritiva e procura, por meio da "história da evolução" do desenho de uma das tarefas que compuseram o produto educacional da dissertação supracitada, evidenciar seu processo de delineamento (elaboração, fases de aplicação e refinamentos).

Para tanto, como ponto de partida, em uma próxima seção, apresentamos uma breve discussão de limite de uma sequência numérica e das características do ambiente de aprendizagem desenvolvido no processo de delineamento das tarefas que visam minimizar

\footnotetext{
${ }^{1}$ Traduções usualmente encontradas para developmental research, design research ou design-based research.

2 Em Ramos e Trevisan (2017), produto educacional resultante da dissertação da primeira autora, está disponibilizado o conjunto completo de tarefas, cuja caracterização é apresentada na continuidade deste texto.
} 
obstáculos relacionados à compreensão desse conceito, levando em conta ideias incompletas ou equivocadas que os estudantes apresentam. Em seguida, vêm os aspectos dos procedimentos metodológicos considerados no desenvolvimento da pesquisa, em particular da tarefa destacada. Subsidiada na construção teórica, apresentamos uma descrição e discussão do processo de delineamento da tarefa selecionada. Encerramos com as considerações finais, seguidas das referências bibliográficas.

\section{Referencial teórico}

Apresentamos nesta seção o referencial teórico que sustenta tanto a dimensão de conteúdo (estudo de limites de sequências numéricas) quanto à dimensão pedagógica (o trabalho por meio de episódios de resolução de tarefas) da proposta.

\section{Limite de uma sequência numérica}

As dificuldades apresentadas por estudantes de CDI na compreensão de conceitos centrais da disciplina (Limite, Derivada e Integral de funções reais de variável (is) real (is)) são temas presentes em pesquisas de Educação Matemática há algumas décadas. Em particular, destacamos o interesse por aquelas que têm estreita ligação com o conceito de limite de sequências numéricas. Em geral, essas dificuldades estão relacionadas ao não entendimento do que seja uma sequência e de ideias subjacentes ao conceito de seu comportamento no infinito, em especial, o conceito de limite.

Ideias incompletas e, em alguns casos, errôneas que são apresentadas por estudantes sobre o conceito de limite devem-se a não conceberem processos infinitos, restringindo-se a finitos (ROH, 2008). Nessa mesma direção, Santos (2005) afirma que estudantes, mesmo aqueles que já cursaram CDI, associam a definição formal de limite a um movimento físico de "aproximação" finito, enquanto, na comunidade científica, o conceito é concebido de forma estática, obtido por um caminho algébrico.

Przenioslo (2005) relata que o entendimento de uma sequência como regra que produz números; conjunto de termos que obedecem regras; longa lista de números, bem como a crença de que sequências numéricas infinitas não possíveis de serem observadas por envolverem um número ilimitado de termos podem ser entraves para entender conceito de limite

Envolvidos no processo de elaborar uma compreensão do conceito de limite, Kumsa, Petterson e Andrews (2017) relacionam obstáculos de três naturezas: epistemológica; cognitiva; didática. Obstáculos de natureza epistemológica estão relacionados ao próprio conceito (devido a outros conceitos utilizados em sua definição formal, por exemplo, infinito, incremento). Os de natureza cognitiva estão relacionados ao processo de aquisição do 
conceito (Por exemplo, diferenças entre o conceito-imagem apresentado pelo estudante e a definição formal de limite). Os de natureza didática estão relacionados ao modo como o conceito é desenvolvido no ambiente de ensino e de aprendizagem.

Acerca do último aspecto, foco de nosso interesse, Przenioslo (2005) destaca que uma abordagem de ensino, que visa minimizar obstáculos relacionados à compreensão do conceito de limite de uma função, deve levar em conta ideias incompletas ou equivocadas sobre limites de uma sequência, tais como: (i) o limite existe se os termos aproximam-se de um ponto, mas, não o atingem; (ii) uma sequência convergente é monótona; (iii) se um número infinito de termos de uma sequência aproximam-se de um valor, então a sequência é convergente. Para o autor, tais ideias podem ser fruto de abordagens didáticas que apresentam precocemente uma definição formal de limite de uma função.

Em um ensino tradicional de CDI, práticas que focam no desenvolvimento de procedimentos matemáticos são corriqueiras, por exemplo, fatoração de polinômios, ao estudar limites. Segundo Nunes (2001), esses procedimentos são enfatizados, em vez de discussões mais teóricas, devido à dificuldade em dar significado ao conceito. Weigand (2014) aponta que, muitas vezes, os estudantes até conseguem calcular limites, mas apresentam dificuldades referentes à compreensão de uma definição formal, já que "não são capazes de utilizar a definição adequadamente em um dado contexto, ou não são capazes de resolver problemas em um nível formal” (WEIGAND, 2014, p. 603, tradução nossa).

Esse autor defende uma revitalização do tema Sequências Numéricas na disciplina inicial de CDI; pois considera que as sequências são objetos matemáticos diretamente relacionados ao conceito de limite, e fundamentais para o desenvolvimento/elaboração de outros conceitos do CDI, como séries infinitas, continuidade de uma função, derivada e integral (WEIGAND, 2004; 2014).

Em seus trabalhos, Weigand $(2004 ; 2014)$ destaca que o estudo de limites de uma função deve ser precedido pelo trabalho com sequências numéricas, uma vez que (i) são inúmeros os problemas matemáticos que podem ser representados por sequências; (ii) que sequências são ferramentas que possibilitam o desenvolvimentos de conceitos necessários à compreensão de processos contínuos e (iii) que o entendimento do conceito de sequência pode auxiliar no desenvolvimentos de ideias centrais do CDI, como derivadas e integrais. Sobre este último ponto, o autor apresenta uma proposta de trabalho que temos adotado em nossas aulas de CDI 1, na qual a exploração de sequências de diferenças e somas parciais antecedam estudo de limites, derivadas e integrais propriamente, pois fornecem uma "base intuitiva" para sua compreensão. 


\section{Episódios de resolução de tarefas em aulas de CDI}

A abordagem que defendemos para nossas aulas de CDI é a organização de "ambientes de aprendizagem pautados em episódios de resolução de tarefas" (COUTO et al., 2017; TREVISAN; MENDES, 2018), expressão adaptada de shift problem lessons, proposta por Palha et al. e Van Hout-Wolters (2013) e Palha et al. (2015). A organização de tarefas que integrem um ambiente educacional para o CDI é um dos objetivos do projeto ${ }^{3}$ do qual este trabalho é recorte.

O termo tarefa é utilizado para descrever uma ampla variedade de atividades destinadas à aprendizagem matemática, havendo uma diversidade de quadros teóricos analíticos que tratam do tema. Entendemos tarefa como "o amplo espectro composto por 'coisas a fazer' pelos estudantes em sala de aula, o que inclui desde a execução de exercícios algorítmicos até a realização de investigações ou construção de modelos matemáticos" (TREVISAN et al., 2015, p. 3). Tal definição é alinhada àquela proposta por Pochulu et al. (2013, p. 4999, tradução nossa), que tomam tarefas como "situações que o professor propõe (problema, investigação, exercício, etc) na aula, e estas são o ponto de partida da atividade do estudante, que, por sua vez, produz como resultado sua aprendizagem". Assim, destaca-se o fato de que, dependendo de suas características, as tarefas podem (ou não) tornar-se fonte de oportunidade para a aprendizagem dos estudantes.

Por um lado, o CDI presente em salas de aulas regulares é apresentado como um assunto pronto e acabado, na forma final da teoria. Geralmente, são apresentadas definições, regras e algoritmos, e se espera que os alunos as reproduzam (FREUDENTHAL, 1973, 1991). Por outro, pesquisas desenvolvidas no âmbito da Educação e da Educação Matemática há algumas décadas apontam que abordagens de ensino promissoras devem oportunizar aos estudantes trabalhar de forma colaborativa, com tarefas de caráter investigativo e que integrem recursos tecnológicos, propiciem a reflexão e promovam a interação. De forma quase parodoxal, Stingler e Hiebert (2004, p. 12) destacam, porém, que, apesar dos avanços dessas pesquisas, a maioria das propostas de reformas no ensino da Matemática "pararam na porta da sala de aula", e esta mudou "muito pouco nos últimos 100 anos".

O trabalho com episódios de resolução de tarefas mostra-se uma proposta factível que busca minimizar essa lacuna, uma vez que as condições reais de ensino em nossas turmas de $\mathrm{CDI}^{4}$ inviabilizam a realização de um trabalho que atenda plenamente, ao longo de todo o curso, pressupostos de tendências para o ensino de Matemática apontado pela Educação Matemática, como a resolução de problemas, a investigação ou a modelagem matemática.

\footnotetext{
3 "Investigação de um ambiente educacional para o Cálculo Diferencial e Integral (CDI) em condições reais de ensino", submetido e aprovado no Edital Universal 14/2014 do CNPq.

${ }^{4}$ Uma análise detalhada dessas condições reais é apresentada em Ramos, Fonseca e Trevisan (2016).
} 
Tais episódios constituem momentos do curso nos quais os estudantes são convidados a explorar, intuitivamente, conceitos, elaborar conjecturas, testá-las e compartilhar com seus colegas suas conclusões.

Um dos pressupostos dessa forma de trabalho é que as tarefas não sejam precedidas da apresentação de conceitos, mas que permitam ao grupo de estudantes explorá-las intuitivamente, sem que uma definição ou conceito seja previamente apresentado; em seguida, por meio das intervenções do professor e da discussão no grupo, os estudantes são incentivados a "refinar" os conceitos subjacentes (construindo, sempre que possível, uma definição provisória - TREVISAN, MENDES, 2017); por fim, a partir da sistematização coletiva mediada pelo professor, elabora-se uma definição formal (que, muitas vezes, ainda é restrita a casos particulares, mas é revisada e ampliada ao longo do curso).

A Figura 1 apresenta um esquema que relaciona elementos da estrutura de um processo de aprendizagem pautado em episódios de resolução de tarefas, discutidos em detalhes por Ramos (2017).

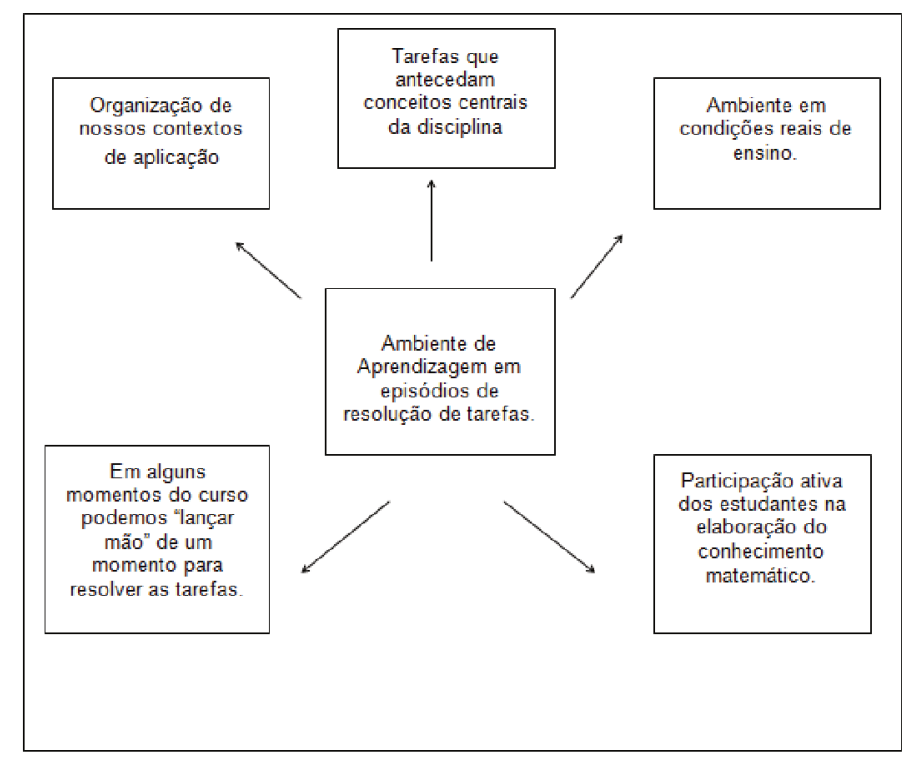

Figura 1 - Elementos de um processo de aprendizagem pautado em episódios de resolução de tarefas.

Fonte: Ramos (2017, p. 33)

Nesse ambiente, o professor assume o papel de “incentivador" de estratégias, não cabendo a ele, durante a exploração da tarefa, fornecer respostas, mas sim apresentar aos grupos indagações que os auxiliem pensar estratégias, desenvolver procedimentos, elaborar e testar conjecturas e, sempre que possível, mediar a sistematização de conceitos subjacentes.

\section{Procedimentos metodológicos}

\section{Caracterização da pesquisa}

Nossa pesquisa é de cunho qualitativo e, em função de seu caráter cíclico, adota pressupostos da IBD. A questão fundamental é a descrição detalhada e a interpretação de todo 
o processo, do que aconteceu, não sendo definida de antemão a busca de um "resultado final".

Nesse sentido,

a existência de um produto final que preencha as exigências de um trabalho competente depende muito mais da forma como o estudo é executado e da facilidade relativa à expressão conceptual e à escrita parte do investigador do que da especificidade do plano de investigação. Não admira que os avaliadores de propostas qualitativas tendam a dar muito peso ao trabalho anterior de autores para a avaliação das possibilidades de sucesso do projeto proposto (BOGDAN, BIKLEN, 1994, p. 107).

A IBD, conforme apontam Barbosa e Oliveira (2015),

[...] refere-se àquelas investigações que envolvem delineamento, desenvolvimento e avaliação de artefatos para serem utilizados na abordagem de um determinado problema, à medida que se busca compreender/explicar suas características, usos e/ou repercussões (BARBOSA, OLIVEIRA, 2015, p. 527).

Nesse tipo de pesquisa,

os alunos são testados continuamente durante o experimento. Se a aprendizagem observada é diferente da esperada, isso implica que as atividades de aprendizagem têm que ser mudadas durante ou depois de uma aula, e que as hipóteses têm de ser adaptadas para a nova situação (EERDE, 2013, p. 3).

Mckenney e Reeves (2012 apud MATTA et al., 2014) destacam cinco característicasda IBD, que reconhecemos em nossa pesquisa:

i) teoricamente orientada - As teorias são tomadas como pontos de partida, de chegada e de investigação na IBD. Nossa conjectura de ensino, a ser apresentada na continuidade deste texto, é construída com respaldo no referencial teórico apresentado na seção anterior;

ii) intervencionista - Utilizamo-nos do fundamento teórico escolhido, em diálogo com nosso contexto de aplicação (detalhado a seguir), para que a pesquisa desenvolvesse uma aplicação que irá intervir no campo da prática didática, no intuito de produzir um material didático (representado por um caderno de tarefas);

iii) colaborativa - Nossa investigação incluiu, além da pesquisadora e seu orientador, um grupo de pesquisa constituído por outros estudantes (mestrandos do PPGMAT e estudantes de graduação) e docentes do PPGMAT, no intuito de "mergulhar no estudo e entendimento do contexto a ser pesquisado, e assim ganh[ar] a capacidade de dialogar e de estarem engajados no problema" (MATTA et al., 2014, p. 26);

iv) fundamentalmente responsiva- Nossa IBD foi "moldada pelo diálogo entre a sabedoria dos participantes [acima citados], o conhecimento teórico [descrito anteriormente neste texto], suas interpretações e advindos da literatura, e pelo conjunto dos testes e validações diversas realizadas em campo" (MATTA et al., 2014, p. 27);

v) iterativa - Nossa IBD intenta a "construção de soluções práticas", visto que considera condições reais de ensino de CDI. 
Tal abordagem procura entrelaçar ciclos de elaboração de materiais educacionais ao desenvolvimento de uma teoria de ensino por eles subsidiada, envolvendo ciclos de aplicação, análise, avaliação e validação como mostrado na Figura 2.

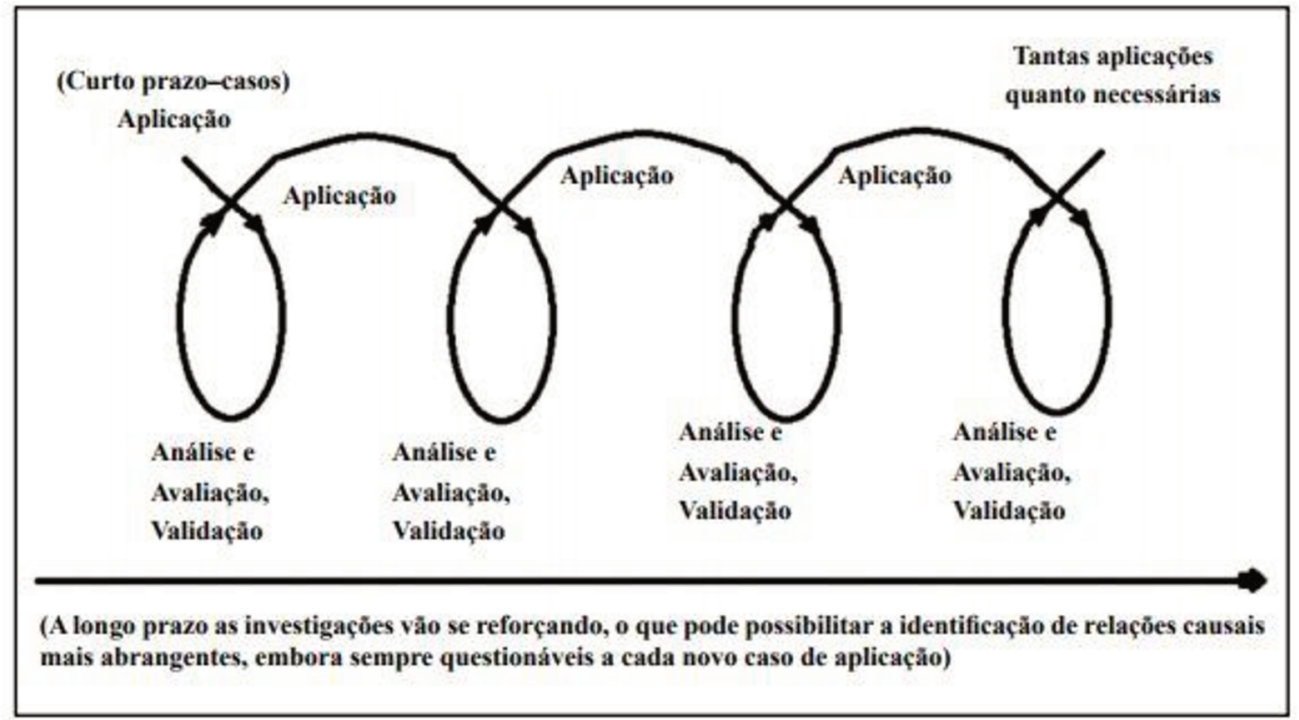

Figura 2 - Ciclos do Design- Based Research

Fonte: Matta et al. (2014)

Molina, Castro e Castro (2007) apresentam os experimentos de ensino como modalidades particulares da pesquisa de desenvolvimento. $O$ desenho de tais experimentos contempla o desenvolvimento de processos de planejamento e ensino, assim como a investigação de tais processos em um contexto educacional (a sala de aula). Sua análise ocorre tanto no decurso da implementação da proposta (quando as tarefas são formuladas e aplicadas - o que dificulta uma análise sistemática) quanto após o término da intervenção (análise retrospectiva).

Por meio de um experimento de ensino, os papéis de pesquisador e professor são "aproximados" e se inter-relacionam. Mestre e Oliveira (2016) descrevem o experimento de ensino como uma metodologia que tem o potencial de fazer uma ponte entre a teoria e a prática, a qual integra uma sequência de episódios, um professor/pesquisador e os estudantes visando à compreensão do processo de ensino e aprendizagem. Um fator importante, que emerge do experimento de ensino, segundo Mestre e Oliveira (2016), é o papel do professorinvestigador que contempla a avaliação do processo como um todo e o raciocínio dos estudantes, podendo interferir nos episódios com vistas a subsidiar todo o processo. Em nosso contexto, assumimos tal papel e podemos ressaltar que o estar inserido em nossa pesquisa, tanto como professor quanto como pesquisador, contribuiu para o seu desenvolvimento. 


\section{$O$ contexto da pesquisa e a coleta de dados}

O contexto do qual provêm os dados que subsidiam o processo de construção do artefato (produto educacional) inclui estudantes de Engenharia que cursaram CDI em turmas sob a responsabilidade dos autores. Realizamos ciclos de aplicação que ocorreram em quatro momentos, ao longo dos anos de 2016 e 2017, em quatro turmas de CDI 1 (a análise de elementos que resultaram dessa opção metodológica é parte da análise).

Nossa experiência de ensino tem como contexto dois campi de uma universidade federal do estado do Paraná. Trata-se de um contexto real de ensino: uma sala com 50 estudantes e suas possíveis dificuldades com os conceitos trabalhados na disciplina, o desinteresse por parte de alguns, um plano de ensino a cumprir, avaliações, correções, enfim, tudo o que realmente compõe o dia a dia de sala de aula. Uma descrição detalhada desse contexto e de suas condições reais foi feita de forma detalhada por Ramos, Fonseca e Trevisan (2016), e alguns elementos mais relevantes são destacados a seguir.

As turmas iniciam-se com 44 alunos regulares, além de 6 a 8 estudantes na modalidade especial ${ }^{5}$ (alunos que não obtiveram aprovação anterior na disciplina). A seleção dos estudantes ocorre pelo Sistema de Seleção Unificado (SISU/MEC). O Sisu é o sistema informatizado, gerenciado pelo Ministério da Educação ( $\mathrm{Mec}$ ), por meio do qual as instituições públicas de educação superior oferecem vagas a candidatos participantes do Exame Nacional do Ensino Médio (Enem). A seleção acontece duas vezes ao ano, no primeiro e no segundo semestres. Muitos alunos ingressam no curso de engenharia mesmo não sendo o pretendido inicialmente e buscam posteriormente a transferência interna e/ou externa para o curso almejado, fazendo com que haja um número significativo de desistências ao longo do semestre. Em termos de infraestrutura, as salas de aulas seguem a estrutura usual, quadro-negro, mesa do professor, carteiras dos alunos em disposição de filas e data show.

A disciplina de CDI 1 é cursada no $1^{\circ}$ semestre e possui uma carga de 90 horas-aula. Sua ementa contempla o estudo de funções, limites, derivadas e integrais de funções reais, de uma variável real, que organizamos segundo uma estrutura curricular "não usual" (TREVISAN; MENDES, 2017), conteúdos em formato de espiral. Assim, embora formalmente o estudo de sequências numéricas e sua convergência não façam parte da ementa da disciplina, temos optado por explorá-lo nas aulas iniciais, visto que o estudo de sequências de diferenças (TREVISAN et al., 2018) e da variação acumulada leva a definições provisórias de derivada e integral, respectivamente (que ocorrem ainda no primeiro mês de aula).

\footnotetext{
${ }^{5} \mathrm{Em}$ geral, alunos que foram reprovados com média inferior a 4,0 e/ou frequência inferior a 75\%. Aos alunos reprovados com média superior a 4,0 (e inferior a 6,0 ), e que apresentaram pelo menos $75 \%$ de frequência nas aulas, é permita a matrícula em turma sem presença obrigatória, ou seja, não precisam assistir às aulas, somente realizarem as avaliações.
} 
Em nosso problema de trabalho em episódios de resolução de tarefas, temos dedicado cerca de 25 horas do curso (cerca de 10 encontros de 3 horas-aula de 50 minutos) a esses episódios, planejados para anteceder o estudo "formal" dos conceitos de função de uma variável real, limite, derivada e integral. As tarefas que compõem a pesquisa foram aplicadas em 6 dias de aula (18 h/a).

Em todas as aplicações, os estudantes foram organizados em grupos de três a quatro, totalizando uma média de 14 grupos em cada turma, o que possibilitou a troca de ideias na elaboração de estratégias para a resolução de cada tarefa proposta. Para a coleta de dados, utilizamos a produção escrita dos estudantes, gravações de áudios e vídeo durante o trabalho com as tarefas.

Visto que a pesquisa é realizada em condições reais de ensino, elencamos algumas limitações do âmbito da coleta (que não comprometeram os resultados obtidos).

- Nem sempre a aplicação da tarefa ocorreu como o planejado. Em função do tempo disponível para a realização da tarefa, muitas vezes não foi possível permitir que as equipes explorassem exaustivamente a tarefa. Em geral, buscava-se organizar o tempo, de modo que, em um mesmo encontro, houvesse tempo para resolução, discussão e sistematização da tarefa.

- As condições materiais necessárias à aplicação, como planejado, muitas vezes tinham que ser adaptadas, seja porque poucos alunos - ou quase nenhum - levavam o computador para a aula (embora houvesse um combinado quanto a isso e a tarefa exigisse sua utilização), às vezes o projetor falhava; às vezes alunos chegavam atrasados ou saíam durante a aula;

- Nem sempre a coleta de dados ocorria como planejada, às vezes a gravação ficava inaudível; outras, o gravador ou a câmera falhava; algumas equipes não entregaram a produção escrita e, quando solicitado na aula seguinte, essa produção não foi encontrada.

- Algumas tarefas não eram finalizadas em uma aula, mas, na aula, seguinte nem todos estavam presentes, mudando a configuração dos grupos na continuidade do trabalho.

\section{A conjectura para a Experiência de Ensino}

Respaldados nos trabalhos de Przenioslo (2005), Santos (2005), Roh (2008) e Weigand (2014), nossa intenção é a proposição de tarefas que oportunizem aos estudantes a exploração de ideias necessárias à compreensão do conceito de sequências numéricas e sua convergência, em uma abordagem didática que anteceda o estudo de limite de funções. Em outras palavras, que a exploração dessas tarefas envolvendo sequências numéricas seja desencadeadora do ensino de limite de funções reais de variável real. 
Nossa conjectura é que um trabalho sistemático na sala de aula, devidamente orientado a partir de episódios de resolução de tarefas, possibilita aos estudantes explorar de forma intuitiva "ideias básicas" necessárias à compreensão do conceito de limite de uma sequência numérica infinita, das quais listamos as que seguem.

(i) Diferença entre os termos da sequência - Ao investigar a variação entre os termos consecutivos de uma sequência, é possível, no caso de convergente para um número real, construir uma sequência convergente para zero. Ainda é possível analisar que esse comportamento depende exclusivamente de $n$ suficientemente grande, ou seja, a convergência de uma sequência independe do comportamento dos seus $n$ primeiros termos.

(ii) Monotonicidade e limitação - A convergência de uma sequência pode ser garantida se ela for monótona e limitada, entretanto esses comportamentos observados isoladamente não garantem a convergência. Também é possível analisar que toda sequência convergente é limitada, podendo seus limitantes superior e inferior serem ambos distintos do limite da sequência.

(iii) Comportamento em longo prazo - Analisar o comportamento de uma sequência para valores de $n$ "tão grandes quanto quisermos", ressaltando que esse comportamento pode independer do comportamento de seus $n$ termos; assim como o processo estimativo de convergência pode não ser coerente ao processo de obter o limite (comportamento no infinito).

(iv) O limite é um valor que pertence ou não ao conjunto imagem da sequência.

(v) Subsequências com comportamento diferente da sequência - Uma sequência que possui subsequências com comportamentos que convergem para valores distintos são divergentes, discussão da unicidade de limite, a natureza ilimitada do infinito. O processo é provar a convergência, a partir de suas subsequências enquanto um processo paradoxal, já que é impossível escrever todas as suas subsequências.

A exploração dessas ideias resultou em um conjunto de tarefas, organizado na forma de um caderno (RAMOS; TREVISAN, 2017), que compõe o produto educacional resultante da dissertação da primeira autora. No Quadro 1, apresentamos uma breve descrição de cada uma das tarefas que compõem o material, em termos de conteúdos matemáticos e dos objetivos de aprendizagem a eles atrelados, além do número de itens de cada tarefa. 
Quadro 1 - Tarefas para exploração de ideias necessárias à compreensão do conceito de limite de uma sequência numérica infinita

\begin{tabular}{|c|c|l|c|}
\hline Nome da Tarefa & Conteúdo matemático & \multicolumn{1}{c|}{ Objetivo } & $\begin{array}{c}\text { Itens que } \\
\text { compõem a } \\
\text { tarefa }\end{array}$ \\
\hline O caso compunet & $\begin{array}{c}\text { Definição de uma sequência } \\
\text { numérica e estudo de } \\
\text { sequências particulares. }\end{array}$ & $\begin{array}{l}\text { Reconhecer diferentes tipos de sequência e } \\
\text { seu comportamento. }\end{array}$ & 1 \\
\hline $\begin{array}{c}\text { Diferentes } \\
\text { comportamentos de uma } \\
\text { sequência }\end{array}$ & $\begin{array}{c}\text { Tipos sequências e critérios de } \\
\text { convergência }\end{array}$ & $\begin{array}{l}\text { - Estabelecer relação entre diferentes } \\
\text { comportamentos de uma sequência } \\
\text { numérica. } \\
\text { Elaborar definição provisória de } \\
\text { ncia de uma sequência }\end{array}$ & 4 \\
\hline $\begin{array}{c}\text { Convergência de uma } \\
\text { sequência numérica }\end{array}$ & $\begin{array}{c}\text { Convergência de uma } \\
\text { sequência numérica: definição } \\
\text { formal }\end{array}$ & $\begin{array}{l}\text { Elaborar definição formal de } \\
\text { convergência de uma sequência }\end{array}$ & 4 \\
\hline
\end{tabular}

Fonte: Ramos e Trevisan (2017).

\section{Apresentação dos dados}

Uma IBD procura entrelaçar a elaboração de materiais educacionais ao desenvolvimento da teoria de ensino por ele subsidiada (aqui representada por um conjunto de ideias subjacentes ao conceito de convergência de uma sequência numérica). Conforme as nossas opções metodológicas, buscamos, mediante a elaboração de nossa sequência de tarefas para um ambiente de aprendizagem em condições reais de ensino de CDI, refletir acerca do papel das tarefas propostas na aprendizagem de conceitos. Ao mesmo tempo, buscamos considerar tarefas que possam ser trabalhadas em salas de aulas regulares do curso: de modo restrito, o item 2 da segunda tarefa que compõe o produto educacional já descrito (Quadro 1).

Assim, em nossa análise, por meio do item da tarefa selecionada, procuramos evidenciar (i) elementos resultantes do percurso metodológico de delineamento das tarefas; (ii) fases de seu delineamento; (iii) análise retrospectiva e considerações da pesquisadora (apresentadas como considerações finais do artigo).

(i) Elementos do percurso metodológico

No processo de delineamento das tarefas, realizamos ciclos de aplicação que ocorreram em quatro momentos ao longo dos anos 2016 e 2017, em quatro turmas de CDI 1. No segundo semestre de 2016, foram realizadas experiência de ensino em duas turmas: (i) na turma do orientador, em que a aplicação foi conduzida em conjunto pela pesquisadora e pelo docente responsável pela turma (contexto A); (ii) na turma da primeira autora, que assume o duplo papel de pesquisadora e docente da turma (contexto B), sendo este o contexto selecionado para a apresentação e a análise de dados em sua dissertação (RAMOS, 2017). 
Essa dinâmica também ocorreu no primeiro semestre de 2017; porém, diferentemente do ocorrido no segundo semestre de 2016, em 2017 a autora não participou diretamente da aplicação na turma de seu orientador (contexto C), mas conduziu novamente o trabalho em sua turma (contexto D). Ambos reaplicaram as tarefas em suas turmas, de forma independente, no intuito apenas de refinar a constituição da versão final do produto educacional. O esquema mostrado na Figura 3, cujo detalhamento é apresentado por Ramos (2017), ilustra esses contextos. No esquema é possível seguir o caminho do delineamento das tarefas a partir da direção das setas. O contexto B, destacado no esquema, foi objetivo de análise de Ramos (2017).

Em função de nossas opçốes metodológicas, o caráter cíclico da IBD resultou em um desenho peculiar, no qual os ciclos de aplicação, análise, avaliação e validação foram entrelaçados, oferecendo assim mais "robustez" à proposta (MATTA et al., 2014), se comparado ao desenho "padrão" proposto na Figura 2.

Em nossas quatro aplicações, o entrelaçamento do contexto A (que serviu como antecipação) com o contexto B (que promoveu uma primeira análise e avaliação) gerou um redesenho das tarefas. Essas, quando aplicadas no contex to $\mathrm{C}$ e D, promoveram um novo ciclo de análise e avaliação, validado no contexto $\mathrm{D}$, culminando na proposta final do produto educacional. Tal dinâmica é ilustrada no esquema apresentado na Figura 4. 


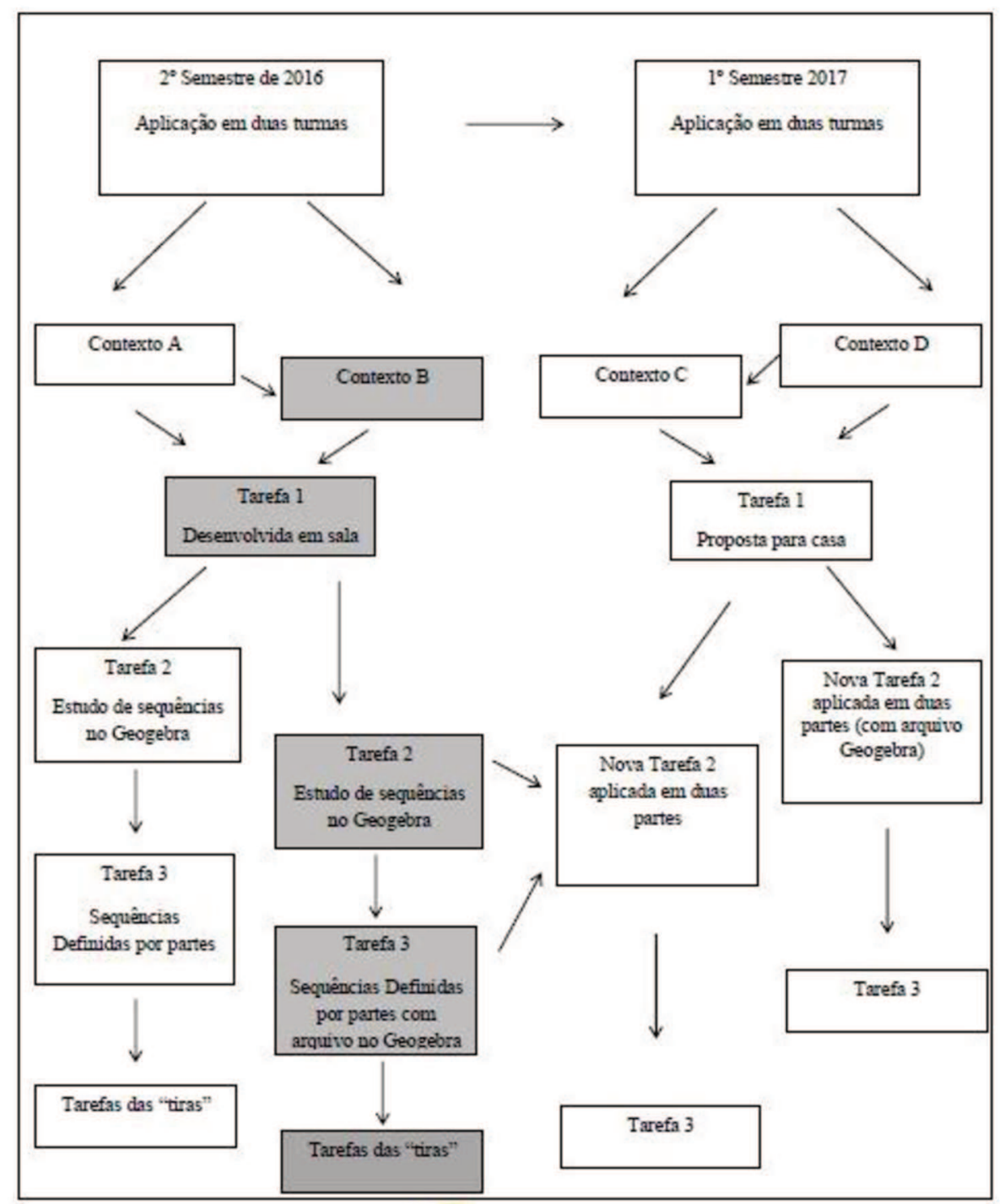

Figura 3 - Mapa das Tarefas Fonte: autores.

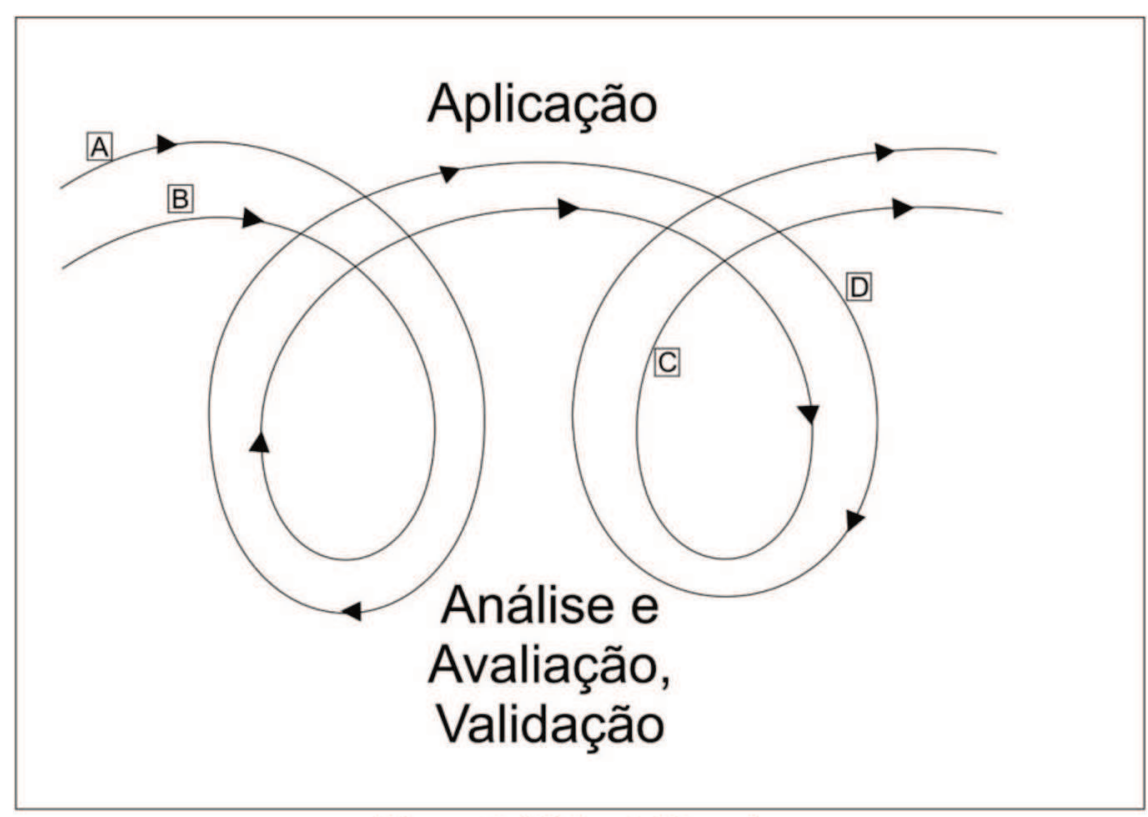

Figura 4- Ciclos da Pesquisa

Fonte: autores 
(ii) Fases de delineamento de uma das tarefas

A primeira etapa do processo de delineamento consistiu em propor aos estudantes do contexto A que apresentassem, individualmente, uma explicação sobre o que entendiam por sequência numérica, assim como cinco exemplos. O objetivo foi identificar suas concepções prévias acerca desse conceito matemático, o que eles entendiam e se conseguiam exemplificar segundo o significado por eles atribuído, bem como analisar os modos pelos quais os estudantes representavam uma sequência numérica.

Do material coletado, identificamos respostas que contemplam tanto caracterizações informais (por exemplo, "algo que segue um determinado padrão", ou "uma sucessão de fatos, números") quanto, matemáticas ("função cujo domínio é o conjunto dos números inteiros positivos"). Quanto aos tipos de representação utilizados, tanto na definição quanto nos exemplos, há uma prevalência das linguagens verbal e numérica e, em menor grau, algébrica (nesse caso, prioritariamente por meio da expressão do termo geral) e gráfica. Em relação aos exemplos apresentados, em geral, são associados a casos particulares, como progressões aritméticas ou geométricas, ou sequência de Fibonacci.

Dessa análise inicial, algumas conjecturas foram levantadas no que tange à elaboração de tarefas. Com vistas a ampliar e aprofundar sua compreensão sobre o objeto matemático sequência numérica, era necessário oportunizar aos estudantes

(i) lidar com situações que permitissem articular diferentes representações de uma sequência numérica (verbal, numérica, gráfica e algébrica - tanto pela expressão do seu termo geral quanto pela definição recursiva), bem como atribuir significado à sua definição matemática (organizando, para isso, uma primeira tarefa, "O caso compunet" - Quadro 1).

(ii) explorar, de forma intuitiva, o comportamento a longo prazo de sequências (incluindo aqui casos de convergência, tanto com limite zero quanto diferente de zero, e de divergência), visando elaborar uma definição provisória de sequência convergente ${ }^{6}$.

Para oportunizar os aspectos apresentados no item (ii), acima, foi elaborado um primeiro desenho de tarefa, ilustrado na Figura 5 e proposto no contexto B, que envolveu analisar o comportamento de algumas sequências (adaptadas do trabalho de Santos (2005)). A tarefa foi precedida de um momento de aula, no qual foram desenvolvidas representações para

\footnotetext{
${ }^{6}$ Temos trabalhado, em um projeto maior, com a criação de tarefas (e, intrinsecamente associado a isso, investigado formas de utilizá-las em sala de aula, bem como aprendizagens por ela propiciadas) que oportunizem aos estudantes reinventar CDI, que permitam a criação de conceitos e teoremas fundamentais utilizados intuitivamente (por meio da organização de definições provisórias) antes que sejam descritos com precisão ou provadas (TREVISAN; MENDES, 2017).
} 
o termo geral de uma sequência e sua representação recursiva, desenvolvidos também a partir dos softwares Excel e GeoGebra. Após sua resolução, os estudantes foram convidados a explicar o que significava dizer que uma sequência qualquer converge, podendo usar o software nessa análise.

Descreva o que está acontecendo com os termos gerais de cada uma das sequências $\left(a_{n}\right)_{n \in z}$ à
medida que " $n$ assume valores cada vez maiores"
a) $a_{n}=\frac{n+20}{5 n}$
b) $a_{n}=n^{2}$
c) $a_{n}=\frac{n}{n+1}$
d) $a_{n}=(-1)^{n}\left(\frac{n^{2}}{2^{n}}\right)$

Figura 5- tarefa proposta

Fonte: Questão adaptada de Santos (2005).

Nosso intuito, ao construir os itens da tarefa, foi permitir aos estudantes explorar exemplos de sequências com comportamentos diferentes daqueles usualmente observados em progressões aritméticas e geométricas. Assim, colocá-los em contato com exemplos de sequências não monótonas e sequências cujo limite é um valor não nulo, que permitem "colocar em xeque" ideias incompletas ou equivocadas sobre limite de uma sequência citado por Przenioslo (2005). Nossa conjectura era que, ao se deparar com sequências cujo termo geral "fuja" desses casos particulares, os estudantes sentir-se-iam convidados a investigar e inferir algo a respeito do comportamento dessas sequências à medida que " $n$ assume valores cada vez maiores", lançando mão do software Excel ou do GeoGebra para construir uma representação gráfica ou numérica, cuja sintaxe já era conhecida naquele momento.

Porém, observando o modo como os estudantes lidaram com a tarefa, notou-se que uma grande parte deles pôs-se a calcular cada um dos termos da sequência, ocupando-se muito mais em efetuar "cálculos" em vez de tentar inferir algo acerca do comportamento a longo prazo, com base na análise do termo geral de cada sequência. Embora, no GeoGebra, tivesse sido apresentada a sintaxe para a representação gráfica de sequências aos estudantes (detalhado em Ramos e Trevisan (2017)), uma das hipóteses para sua não utilização foi a complexidade ou a falta de segurança quanto a essa utilização.

Tal fato levou-nos a repensar o desenho da tarefa, de modo que o uso do recurso tecnológico ocorresse de forma direcionada, tendo sido aplicada no contexto C. Um arquivo em Geogebra, contendo uma representação gráfica das sequências, cuja imagem é mostrada na Figura 6, foi disponibilizado aos estudantes. Nele, bastava selecionar a sequência desejada (janela de álgebra, à esquerda da tela) e uma representação gráfica era automaticamente gerada (janela de visualização) 


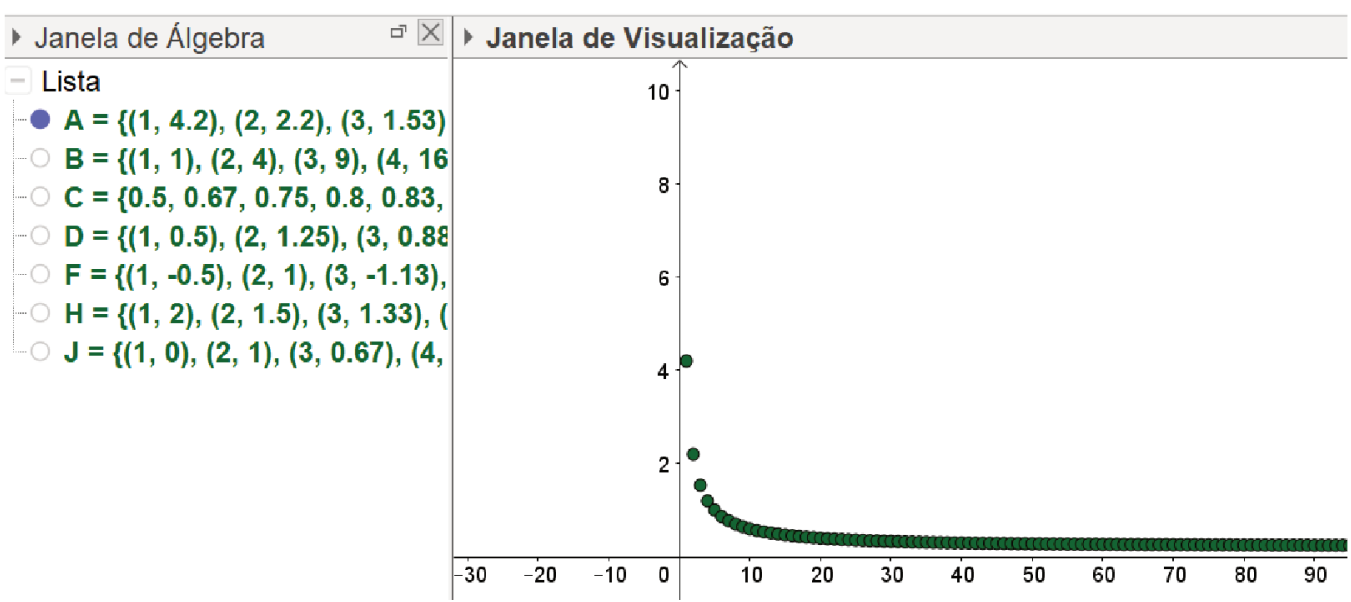

Figura 6 - Imagem da tela no Geogebra.

Fonte: autores.

No contexto $\mathrm{B}$, em que não houve direcionamento quanto ao uso de algum recurso tecnológico, os estudantes analisaram as sequências somente em termos de substituição numérica e esboços gráficos manuais, o que difere das aplicações seguintes nos contextos $\mathrm{C} \mathrm{e}$ $\mathrm{D}$, em que o uso ocorreu de forma direcionada e com a disponibilização do arquivo GeoGebra.

O próprio processo de direcionamento, que, à primeira vista, pode parecer prescritivo, pode ser reconhecido como uma oportunidade do estudante de refletir a respeito do conceito de sequência enquanto uma relação em que, para cada inteiro positivo $n$, existe um número correspondente $a_{n}$. Esse conceito é realçado, pois, para se ter uma representação gráfica de uma sequência, é preciso representar cada um de seus elementos como um par ordenado $\left(n, a_{n}\right)$.

Outro elemento que destacamos é que, em sua aplicação no contexto B, o comportamento da sequência presente no item (a) foi descrito por grande parte dos grupos de estudantes como "convergente para 0" (Figura 7). Já nos contextos C e D, com o auxílio do GeoGebra,o limite da sequência foi identificado pelos estudantes como dois décimos $(0,2)$, haja vista que o software auxiliou na análise da sequência "como um todo" (e não apenas em alguns pontos), possibilitando analisar $a_{n}$ para valores "suficientemente grandes" de $n$. O direcionamento do professor favoreceu aos alunos reconhecer a possibilidade de uma representação gráfica para uma sequência, além da representação tabular explorada no contexto B.

Lidar com esse conceito por meio de uma representação gráfica auxilia o aluno a reconhecer que uma análise de poucos termos (até $n$ ) pode não favorecer a análise de seu comportamento no infinito, assim como favorece ao estudante interpretar o resultado, em aulas tradicionais, obtido a partir de procedimento algébrico de resolver o limite do termo geral de uma sequência, no caso do item (a), como $\lim _{n \rightarrow \infty} a_{n}=\frac{1}{5}$.Em vez de os alunos 
receberem a definição que se $\lim _{n \rightarrow \infty} a_{n}$ existe, dizemos que a sequência converge (ou é convergente), e eles são convidados a reconhecer essa dependência.

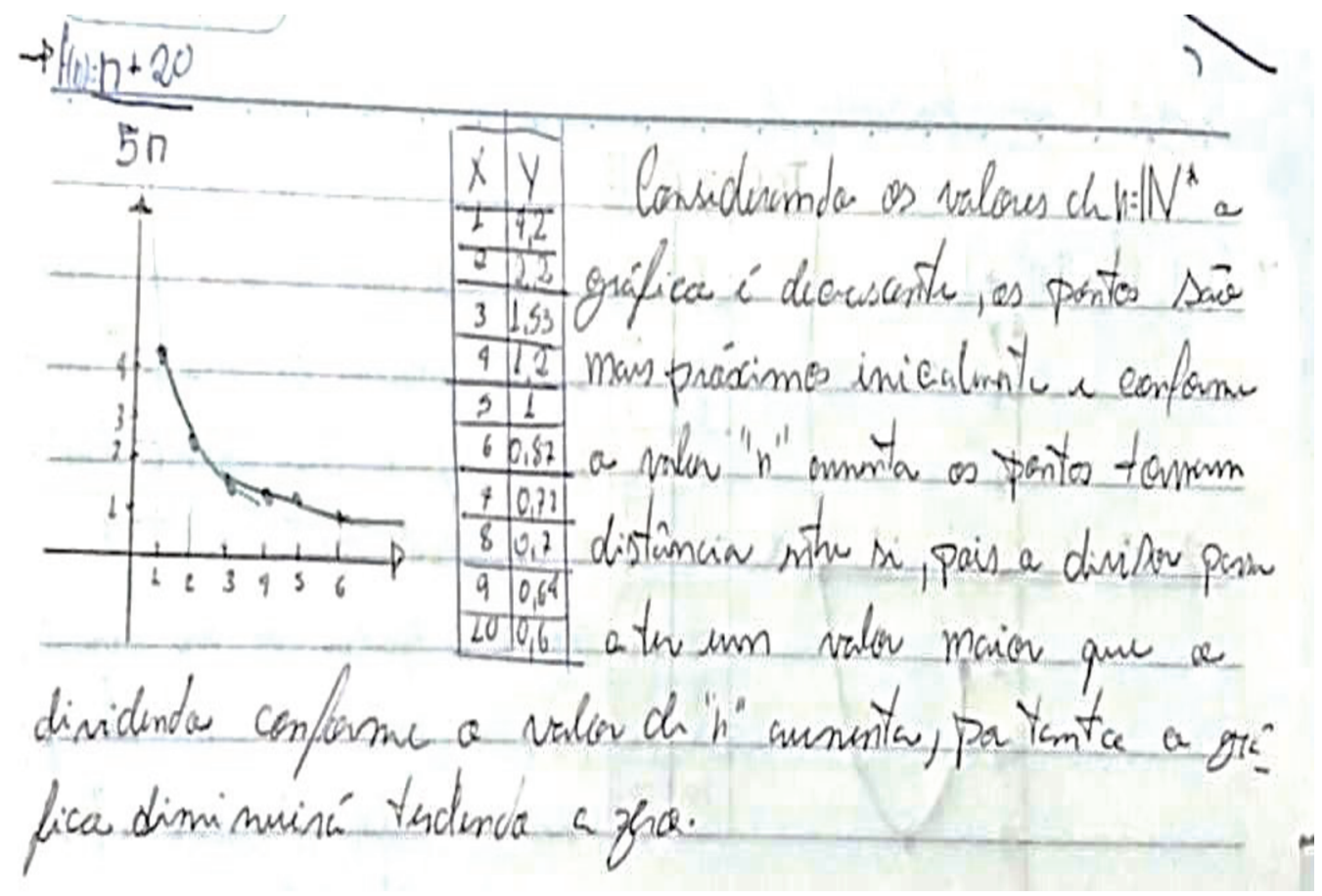

Figura 7- Produção escrita de um grupo que não fez uso do software (Contexto B). Fonte: autores.

Já a sequência presente no item (d) tem um comportamento que permitiu "colocar em xeque" a convergência de sequência intimamente relacionada à monotonicidade. Se uma sequência não é monótona, então ela não converge? As sequências podem apresentar comportamentos distintos, não somente relacionados a monotonicidade e convergência, pois, toda sequência monótona e limitada é convergente, bem como toda sequência convergente é limitada. Mas nem toda sequência limitada é convergente. Tais conclusões foram estabelecidas pelos grupos dos contextos $\mathrm{C}$ e D, ao passo que, no contexto $\mathrm{B}$, a substituição numérica de alguns "poucos valores” levava a conclusões matematicamente incorretas.

De modo geral, a exploração das tarefas nos contextos C e D levaram à problematização de algumas das "ideias básicas" necessárias à compreensão do conceito de limite de uma sequência numérica: (i) diferença entre os termos da sequência; (ii) monotonicidade e (iii) comportamento em longo prazo. Entretanto, aspectos relacionados ao fato de o limite pertencer ou não ao conjunto imagem da sequência, bem como de existirem sequências divergentes e subsquências convergentes, não foram mobilizados a partir dos itens propostos no contexto $\mathrm{C}$, mesmo com a utilização do recurso tecnológico. Assim, a tarefa foi ajustada de modo que, no contexto $\mathrm{D}$, foram incluídos mais itens nos quais tais ideias fossem problemas. Assim, a tarefa foi validada conforme formulação apresentada na Figura 8. 
Descreva o que está acontecendo com os termos de cada uma das sequências $\left(a_{n}\right)_{n \in Z}$ à medida que " $n$ assume valores cada vez maiores"
a) $a_{n}=\frac{n+20}{5 n}$
b) $a_{n}=n^{2}$
c) $a_{n}=\frac{n}{n+1}$
d) $a_{n}=(-1)^{n}\left(\frac{n^{2}}{2^{n}}\right)$
e) $a_{n}=\left\{\begin{array}{cr}1, & e ́ \text { múltiplo de } 10 \\ 1+\frac{1}{n}, & \text { para qualquer outro valor }\end{array}\right.$
f) $f_{n}=\left\{\begin{array}{ccccc}1, & \text { se } & n & e ́ & \text { par } \\ 1-\frac{1}{n}, & \text { se } & n & e ́ & \text { impar }\end{array}\right.$

Figura 8- tarefa proposta

Fonte: Questão adaptada de Santos (2005).

Esse processo de delineamento não se encerra, pois, dentro de um processo de desenvolvimento de um mestrado, é preciso ter um número finito de (re) formulação de enunciados de cada tarefa. Entretanto, a cada implementação em contexto real de sala de aula, seja pelos professores autores deste texto, seja por leitores que se interessarem, o processo se reinicia, e novas considerações podem ser levantadas. É possível, por meio de uma figura de linguagem, reconhecer o delineamento de tarefas em uma sequência, em que o termo geral $a_{n}$ é o desenho da tarefa em seu momento de aplicação $n$, e que tende a uma enunciação que permite ao aluno desenvolver conceitos do currículo de CDI de formulação provisória para definições formais.

\section{Considerações finais}

O recorte de tarefa analisado neste texto traz elementos de uma proposta de delineamento em cursos de CDI 1. Em nossa proposta de trabalho, as tarefas são tomadas como ponto de partida para a elaboração de definições provisórias antes que ocorra uma sistematização de conceito formal. Nesse ambiente, o professor incentiva os estudantes a apresentarem suas estratégias de resolução, pois não buscamos respostas únicas.

O duplo papel de professora-pesquisadora contribuiu para elencar fatos que somente poderiam ser notados por alguém que estivesse convivendo no dia a dia de sala de aula. Toda a interação descrita e analisada contribuiu para a mudança de comportamento dos estudantes nas aulas de CDI 1, no que diz respeito às atitudes de desenvolver conceitos da disciplina. Os alunos, inicialmente, esperavam apresentação e demonstrações de conceitos novos, para depois resolver exercícios de aplicação. Agora se tornaram participantes ativos na elaboração 
de um conceito. Ele não veio pronto. Sua flexibilidade aos expressar estratégias para a sala pôde ser evidenciada. Em consonância com essa perspectiva, Mestre e Oliveira (2016) afirmam que, "ao interagir com os alunos, o professor-investigador procura criar um ambiente de harmonia, motivando-os a explicarem seus raciocínios e ouvindo-os atentamente" (p.28).

A reestruturação na dinâmica do curso, respaldada em Weigand (2014), exigiu uma mudança de atitude no desenvolver das aulas. Antes, seguia-se um plano de ensino usual: um novo conceito era apresentado aos estudantes, demonstraçốes eram apresentadas, exemplos eram trabalhados e, posteriormente, buscavam-se a "fixação" e a "aplicação" com listas de exercícios. Agora o plano de ensino da disciplina é organizado por meio de tarefas e parte das representações dos estudantes apresentadas em suas resoluções, que, sistematizadas, seguem rumo aos conceitos da disciplina, culminando na definição formal.

Essa mudança de dinâmica do curso requereu do professor repensar sua prática de ensino ao desenvolver de conceitos, em especial, requer identificar potencialidades que possam decorrer da aplicação das tarefas e possíveis adaptações, se necessário, para alcançar seu objetivo. Em nosso contexto, partimos das tarefas para o estudo de sequências convergentes como desencadeadoras de limite de uma função. A organização em episódios de resolução de tarefas difere em sua abordagem de uma aula tradicional. As tarefas propostas são o "ponto chave" para a elaboração de conceitos de CDI 1, e os estudantes as desenvolvem em grupos, o que possibilita a troca de estratégias. Nesse sentido, o professor, ao propor uma tarefa, deve identificar conceitos que possam ser primeiramente explorados pelos estudantes de forma intuitiva para, posteriormente, serem sistematizados.

\section{Agradecimentos}

Agradecemos ao CNPq pelo financiamento por meio do Edital Universal 14/2014 (Processo 457765/2014-3).

\section{Referências}

BARBOSA, J. C.; OLIVEIRA, A. M. P. Porque a Pesquisa de Desenvolvimento na Educação Matemática? Perspectivas em Educação Matemática. v. 8, n. 18, p. 527-546, 2015.

BOGDAN, R., BIKLEN, S. Investigação Qualitativa em Educação: uma introdução à teoria e aos métodos. Porto Alegre: Porto Editora, 1994.

COUTO, A. F.; FONSECA, M. O. S.; TREVISAN, A. L. . Aulas de Cálculo Diferencial e Integral organizadas a partir de episódios de resolução de tarefas: um convite à insubordinação criativa. Revista de Ensino de Ciências e Matemática (REnCiMa), v. 8, n. 4, p. 50-61, 2017. 
EERDE, D. van. Design Research: looking into the heart of Mathematics Education. In: THE FIRST SOUTH EAST ASIA DESIGN/DEVELOPMENT RESEARCH. Proceedings... Palembang, 2013.

FREUDENTHAL, H. Mathematics as an Educational Task. Dordrecht: Reidel Publishing Company, 1973.

FREUDENTHAL, H. visiting Mathematics Education. Netherlands: Kluwer Academic Publishers, 1991.

KUMSA, A.; PETTERSSON, K.; ANDREWS, P. Obstacles to students' understanding of the limit concept. In: CONGRESS OF THE EUROPEAN SOCIETY FOR RESEARCH IN MATHEMATICS EDUCATION (CERME), 10. Proceedings... CERME, 10. Dublin, 2017.

MATTA, A. E. R.; DA SILVA, F. P. S.; BOAVENTURA, E. M. Design-based research ou pesquisa de desenvolvimento: metodologia para pesquisa metodologia para pesquisa de desenvolvimento: metodologia para pesquisa aplicada de inovação em educação do século xxi. Revista da FAEEBA - Educação e Contemporaneidade, v. 23, n. 42, p.23-46, 2014.

MESTRE, C. M. M. V. O desenvolvimento do pensamento algébrico de alunos do $4 .^{\circ}$ ano de escolaridade: uma experiência de ensino. 2014. Tese (Doutorado em Educação) Universidade de Lisboa. Lisboa, 2014.

MESTRE, C; OLIVEIRA, H.M. Uma experiência de ensino no4. ${ }^{\circ}$ ano conduzida no duplo papel de professora-investigadora. Quadrante, v. 25, n. 2, p. 25-49, 2016.

MOLINA, M., CASTRO, E.; CASTRO, E. Teaching experiments within design research. The International Journal of Interdisciplinary Social Sciences, v. 2, n. 4, p. 435-440, 2007.

NUNES, M. de N. F. Sequências Numéricas: um Estudo da Convergência através de Atividades. 124.f. Dissertação (Mestrado em Educação Matemática) - Pontifícia Universidade Católica - São Paulo, 2001.

PALHA, S.; DEKKER, R.: GRAVEMEIJER, K.; VAN HOUT-WOLTERS, B. Developing shift problems to foster geometrical proof and understanding. The Journal of Mathematical Behavior. v. 32, n. 2, p. 141-159, 2013.

PALHA, S.; DEKKER, R.: GRAVEMEIJER, K. The effect of shift-problem lessons in the mathematics classroom. Internacional Journal os Science and Mathematics Education. Ministry of Science and Technology, v. 13, n. 6, p. 1589-1623, 2015.

PONTE, J. P.; CARVALHO, R.; MATA-PEREIRA, J.; QUARESMA, M. Investigação baseada em design para compreender e melhorar as práticas educativas. Quadrante, v. 25, n. 2, p. 77-98, 2016.

POCHULU, M; FONT, V.; RODRIGUEZ M. Criterios de diseño de tareas para favorecer el análisis didáctico en la formación de profesores. In: CONGRESSO IBEROAMERICANO DE EDUCAÇÃO MATEMÁTICA, 7. Actas... CIBEM, 7. Montevideo: Uruguai, 2013.

PRZENIOSLO, M. Introducing the concept of convergence of a sequence in secondary school. Educational Studies in Mathematics, v. 60, n. 1, p. 71-93, 2005. 
RAMOS, N. S. Sequências numéricas como desencadeadoras do conceito de convergências: episódios de resolução de tarefas. Dissertação (Mestrado em Ensino de Matemática).

Universidade Tecnológica Federal do Paraná - Londrina, 2017.

RAMOS, N. S.; FONSECA, M. O. S.; TREVISAN, A. L. Ambiente de aprendizagem de cálculo diferencial e integral pautado em episódios de resolução de tarefas. V SIMPÓSIO NACIONAL DE ENSINO DE CIÊNCIA E TECNOLOGIA, Ponta Grossa - PR. Anais... V SINECT: UTFPR, 2016.

ROH, H.K. Students' images and their understanding of definitionsof the limit of a sequence. Educational Studies in Mathematics, v. 69, n. 3, p. 217-233, 2008.

SANTOS, M. G. Um estudo sobre convergência de sequências numéricas com alunos que já tiveram o primeiro contato com a noção de limite. 118.f. Dissertação (Mestrado em Educação Matemática) - Pontifícia Universidade Católica. São Paulo, 2005.

STIGLER, J.; HIEBERT, J. Improving mathematics teaching. Educational Leadership, v.5, n.61, p. 12-16, 2004.

TREVISAN, A. L.; BORSSOI, A.H.; ELIAS, H. R. Delineamento de uma Sequência de Tarefas para um Ambiente Educacional de Cálculo. In: SEMINÁRIO INTERNACIONAL DE PESQUISA EM EDUCAÇÃO MATEMÁTICA, 6. Anais... SIPEM, 6. Brasília: SBEM, 2015.

TREVISAN, A. L.; FONSECA, M. O. S.; PALHA, S. A. G. Aspectos envolvidos na proposição de uma tarefa com uso de recurso tecnológico: análise de uma experiência de ensino em aulas de Cálculo. Revista Diálogo Educacional, v.18, n. 58, p. 713-738, 2018.

TREVISAN, A. L.; MENDES, M. T. Integral antes de derivada? Derivada antes de integral? Limite, no final? Uma proposta para organizar um curso de Cálculo

Integral. Educação Matemática Pesquisa, v. 19, n. 3, p. 353-373, 2017.

TREVISAN; A. L.; MENDES, M. T. Ambientes de ensino e aprendizagem de Cálculo Diferencial e Integral organizados a partir de episódios de resolução de tarefas: uma proposta. Revista Brasileira de Ensino e Tecnologia, v. 11, n. 1, p. 209-227, 2018.

WEIGAND, H.-G. Sequences-basic elements for discrete mathematics. ZDM, n. 36, v.3, p. 91-97, 2004.

WEIGAND, H. G. A discrete approach to the concept of derivative. ZDM, v.4 6, p. 603-619, 2014.

\section{SOBRE OS AUTORES}

NÉLVIA SANTANA RAMOS: Mestra em Ensino de Matemática pela Universidade Tecnológica Federal do Paraná - UTFPR-LD/CP (2017). Professora de Matemática e Física na Educação Básica e no Ensino Superior.

ANDRÉ LUIS TREVISAN: Doutor em Ensino de Ciências e Educação Matemática. Docente do Departamento de Matemática e do Programa de Mestrado Profissional em Ensino de Matemática - Universidade Tecnológica Federal do Paraná (UTFPR).

MARCELE TAVARES MENDES: Doutora em Ensino de Ciências e Educação Matemática. Docente do Departamento de Matemática e do Programa de Mestrado 
Profissional em Ensino de Matemática - Universidade Tecnológica Federal do Paraná (UTFPR).

Recebido: 13 de abril de 2018.

Revisado: 05 de dezembro de 2018.

Aceito: 05 de fevereiro de 2019. 